\title{
Health Risk Behaviors after Disaster Exposure Among Older Adults
}

Sue Anne Bell, PhD, FNP-BC, NHDP-BC ${ }^{1}$, HwaJung Choi, PhD $^{2}$, Kenneth M. Langa, MD, $\mathrm{PhD}^{2,3,4}$, Theodore J. Iwashyna, MD, $\mathrm{PhD}^{2,3,4}$

1.School of Nursing, University of Michigan, Ann Arbor, Michigan USA

2. University of Michigan Medical School, Ann Arbor, Michigan USA

3.Institute for Social Research, University of Michigan, Ann Arbor, Michigan USA

4. Center for Clinical Management Research at VA Ann Arbor Healthcare System, Ann Arbor, Michigan USA

\section{Abstract}

Objective: The aim of this study was to examine the extent to which an exposure to disaster is associated with change in health behaviors.

Methods: Federal disaster declarations were matched at the county-level to self-reported behaviors for participants in the Health and Retirement Study (HRS), 2000-2014. Multivariable logistic regression was used to evaluate the relationship between disaster and change in physical activity, body mass index (BMI), and cigarette smoking.

Results: The sample included 20,671 individuals and 59,450 interviews; 1,451 unique disasters were declared in counties in which HRS respondents lived during the study period. Exposure to disaster was significantly associated with weight gain (unadjusted RRR $=1.19 ; 95 \% \mathrm{CI}$, 1.11-1.27; adjusted RRR $=1.21 ; 95 \% \mathrm{CI}, 1.13-1.30$ ). Vigorous physical activity was significantly lower among those who had experienced a disaster compared to those who had not (unadjusted $\mathrm{OR}=0.89 ; 95 \% \mathrm{CI}, 0.84-0.95$; adjusted $\mathrm{OR}=0.84 ; 95 \% \mathrm{CI}, 0.79-0.89)$. No significant difference in cigarette smoking was found.

Conclusions: This study found an increase in weight gain and decrease in physical activity among older adults after disaster exposure. Adverse health behaviors such as these can contribute to functional decline among older adults.

\section{Keywords}

aging; disaster; health behavior

Correspondence: Sue Anne Bell, PhD, FNP-BC, NHDP-BC, Clinical Associate Professor, School of Nursing, University of Michigan, 400 North Ingalls Rm 2181, Ann Arbor, Michigan 48109 USA, sabell@umich.edu. 


\section{Introduction}

The 2017 hurricane season devastated communities across the United States, putting tens of thousands of older Americans in harm's way, and causing vast damage. While the individual events were dramatic, disasters are not rare; approximately 1,200 federallydeclared disasters occurred in the last decade in the United States. ${ }^{1}$

Disasters, which are by definition stressful and disruptive, can exacerbate health risk behaviors for older adults; thereby, disasters might have both immediate injury risks and enduring health consequences. For example, disproportionate health effects have been documented among older adults in multiple disaster settings, including Hurricanes Sandy (2012), Rita (2005), and Katrina (2005); the Chicago (Illinois USA) Heat Wave of 1995; and the Great East Japan earthquake (2011). ${ }^{2-5}$ While disasters are on the rise, so too is the prevalence of health risk behaviors among older adults. ${ }^{6,7}$ Growing evidence suggests that healthy behaviors can improve, maintain, or assist in regulating health, all of which are especially important in the context of the physical decline that comes along with aging. ${ }^{8}$ Avoiding behaviors such as a sedentary lifestyle, smoking, and weight gain are among the most well-documented for both preventing and also mitigating diseases of aging such as cognitive decline and dementia. ${ }^{9,10}$ Few studies have investigated the association between disasters and health risk behaviors; those that have only looked at a single disaster, not across disasters, limiting generalizability. ${ }^{11,12}$ The hypothesis was that exposure to disaster may contribute to adverse health behaviors beyond the immediate disaster recovery period. In this study, specific health risk behaviors were examined among a sample of disasterexposed older adults, with the purpose of examining the association between exposure to a disaster and change in health risk behaviors for older adults.

\section{Methods}

Disasters, operationalized as weather and climate events with federal disaster declarations, were matched at the county-level to self-reported behaviors for participants aged 55 and above in the Health and Retirement Study (HRS) 2000-2014, a longitudinal panel survey that biannually follows a national sample of Americans over the age of 50 years. ${ }^{13}$ The HRS was approved by the University of Michigan's Institutional Review Board (Ann Arbor, Michigan USA) and is sponsored by the National Institute on Aging (grant U01 AG009740; Bethesda, Maryland USA). Disasters were identified through the Federal Emergency Management Agency's (FEMA; Washington, DC USA) open source data. ${ }^{14}$

Logistic regression was used to evaluate differences in binary outcomes (change in physical activity and cigarette smoking) by disaster exposure status. Multinomial logistic regression was used to assess difference in categorical outcomes (body mass index [BMI] change). These outcomes were selected as they have key relationships to chronic disease onset and progression, and are well-measured longitudinally in the HRS. Further, they represent a range of ease of change-with changing exercise being easier than weight gain, itself easier than changing smoking status. Parameter estimates for logistic regression were exponentiated to odds ratios (OR) and relative risk ratios (RRR), with $95 \%$ confidence intervals $(95 \% \mathrm{CI})$ examined at the $P=.05$ significance level. All analyses were conducted 
in Stata version 14.2 (StataCorp; College Station, Texas USA). Control variables included age, gender, race, ethnicity, marital status, number of household residents, years of education, wealth by quartile, self-reported health status, number of comorbidities, and limitations in activities of daily living. Lagged variables were applied to relevant covariates (marital status, number of household residents, years of education, wealth by quartile, current self-reported health status, number of comorbidities, and activities of daily living) in order to account for values of variables at prior interviews. ${ }^{15,16}$

\section{Results}

The sample included 20,671 individuals and 59,450 interviews. During the study period, 1,451 unique disasters were declared in counties in which HRS respondents lived, ${ }^{1}$ and $60 \%$ of respondents were exposed to one or more of these disasters. Among participants exposed to disaster since the last interview, $46 \%$ reported weight gain compared to $42 \%$ who were not exposed (Figure 1). Using no weight change as the base outcome, exposure to disaster was significantly associated with weight gain (unadjusted RRR $=1.19 ; 95 \%$ CI, 1.11-1.27; adjusted RRR $=1.21 ; 95 \%$ CI, 1.13-1.30; Table 1). Among those exposed to a disaster since the last interview, $41 \%$ reported engaging in vigorous physical activity versus $44 \%$ of those who were not (Figure 1). Both unadjusted and adjusted models showed that vigorous physical activity was significantly lower among those who had experienced a disaster compared to those who had not (unadjusted $\mathrm{OR}=0.89$; $95 \% \mathrm{CI}, 0.84-0.95$; adjusted $\mathrm{OR}=0.84 ; 95 \%$ CI, 0.79-0.89; Table 1).

Little difference in cigarette smoking was found: $12 \%$ of participants who had experienced a disaster since the last interview reported current cigarette smoking compared to $13 \%$ of who had not (Figure 1).

\section{Discussion}

Understanding how disasters affect the long-term health of older adults is a critical need as disasters continue to increase. ${ }^{1}$ In this analysis, significant associations were found between disaster exposure and health risk behaviors, indicating that the acute disruption of a disaster may adversely influence health behaviors, thereby contributing to worse health among older adults. Specifically, vigorous activity, the kind of high-quality physical exercise that is essential for building and retaining muscle strength in older adults, declined among this sample of older adults after disaster. Closely related, older adults exposed to disaster were more likely to gain weight as well, as measured by change in BMI. Cigarette smoking, however, did not have a significant association, a finding that differs from past studies of substance use in individual disasters, ${ }^{12,17}$ perhaps indicating that other habits and social conventions can limit the extent of damage to health behaviors after a disaster. Other studies have examined health risk behaviors among older adults using HRS data; specifically, trends in physical activity, ${ }^{18}$ cigarette use, ${ }^{19}$ and increases in $\mathrm{BMI}^{20}$ among older adults. Only one other study to date has combined HRS data with FEMA disaster declarations. This study found no significant effect size when examining the association between community-level disaster exposure and individual-level changes in disability or the risk of death. ${ }^{21}$ 


\section{Limitations}

This study is unique in that it examines health risk behaviors across numerous types of disasters and geographic locations; however, it is limited by the potential for ecological fallacy, where control variables may interact with affected populations differently-although disaster exposure is properly conceptualized as a community-level variable in this study, not merely an individual-level exposure. This study is further limited by self-report bias, where reporting of health risk behaviors might be associated with disaster exposure and hence influence the estimates. A particular strength of this study is its longitudinal design where changes in health risk behaviors can be observed in association with disaster exposure. This avoids the issue with many cross-sectional studies, which by comparison, cannot address a change in exposure without risking some level of bias.

\section{Conclusions}

This study found an increase in weight gain and decrease in physical activity among older adults after disaster exposure. Adverse health behaviors such as these can contribute to functional decline among older adults. Expanded studies examining trends in health risk behaviors after disaster can better inform interventions to address healthy lifestyles for older adults during the disaster recovery period, moving beyond the traditional focus on immediate injury and psychological trauma.

\section{Abbreviations:}

BMI

FEMA

HRS body mass index

Federal Emergency Management Agency

Health and Retirement Study

\section{References}

1. Federal Emergency Management Agency. Disaster Declarations. 2017. https://www.fema.gov/ disasters. Accessed July 17, 2017.

2. Malik S, Lee DC, Doran KM, et al.Vulnerability of older adults in disasters: emergency department utilization by geriatric patients after Hurricane Sandy. Disaster Med Public Health Prep. 2018;12(2):184-193. [PubMed: 28766475]

3. Cherry KE, Brown JS, Marks LD, et al.Longitudinal assessment of cognitive and psychosocial functioning after Hurricanes Katrina and Rita: exploring disaster impact on middle-aged, older, and oldest-old adults. J Appl Biobehav Res. 2011;16(3-4):187-211. [PubMed: 23526570]

4. Klinenberg EHeat Wave: A Social Autopsy of Disaster in Chicago. Chicago, Illinois USA: University of Chicago Press; 2015.

5. Tanji F, Sugawara Y, Tomata Y, et al.Psychological distress and the incident risk of functional disability in elderly survivors after the Great East Japan Earthquake. J Affect Disord. 2017;221:145150. [PubMed: 28646710]

6. Hill JO, Catenacci V, Wyatt HR. Obesity: overview of an epidemic. Psychiat Clin North Am. 2005;28(1):1-23, vii.

7. Anstey KJ, von Sanden C, Salim A, O'Kearney R. Smoking as a risk factor for dementia and cognitive decline: a meta-analysis of prospective studies. Am J Epidemiol. 2007;166(4):367-378. [PubMed: 17573335] 
8. Whitehead BR. Health behaviors in older adults: considering age, affect, and attitudes. J Health Psychol. 2017;22(13):1652-1657. [PubMed: 26929172]

9. Beydoun MA, Beydoun HA, Wang Y. Obesity and central obesity as risk factors for incident dementia and its subtypes: a systematic review and meta-analysis. Obesity Rev. 2008;9(3):204-218.

10. Lee Y, Back JH, Kim J, et al.Systematic review of health behavioral risks and cognitive health in older adults. Int Psychogeriatr. 2010;22(2):174-187. [PubMed: 19883522]

11. Flory K, Hankin BL, Kloos B, Cheely C, Turecki G. Alcohol and cigarette use and misuse among Hurricane Katrina survivors: psychosocial risk and protective factors. Subst Use Misuse. 2009;44(12):1711-1724. [PubMed: 19895302]

12. Moise IK, Ruiz MO. Hospitalizations for substance abuse disorders before and after Hurricane Katrina: spatial clustering and area-level predictors, New Orleans, 2004 and 2008. Prev Chronic Dis. 2016;13:E145. [PubMed: 27736053]

13. Sonnega A, Faul JD, Ofstedal MB, Langa KM, Phillips JW, Weir DR. Cohort Profile: the Health and Retirement Study (HRS). Int J Epidemiol. 2014;43(2):576-585. [PubMed: 24671021]

14. Federal Emergency Management Agency. OpenFEMA. 2018. https://www.fema.gov/openfema. Accessed January 11, 2018.

15. Bellemare MF, Masaki T, Pepinsky TB. Lagged explanatory variables and the estimation of causal effect. J Politics. 2017;79(3).

16. Dormann C, Griffin MA. Optimal time lags in panel studies. Psychol Methods. 2015;20(4):489505. [PubMed: 26322999]

17. Peters RJ Jr., Meshack A, Amos C, Scott-Gurnell K, Savage C, Ford K. The association of drug use and post-traumatic stress reactions due to Hurricane Ike among Fifth Ward Houstonian youth. J Ethn Subst Abuse. 2010;9(2):143-151. [PubMed: 20509087]

18. Feng X, Croteau K, Kolt GS, Astell-Burt T. Does retirement mean more physical activity? A longitudinal study. BMC Public Health. 2016;16:605. [PubMed: 27439914]

19. Quinones AR, Nagel CL, Newsom JT, Huguet N, Sheridan P, Thielke SM. Racial and ethnic differences in smoking changes after chronic disease diagnosis among middle-aged and older adults in the United States. BMC Geriatr. 2017;17(1):48. [PubMed: 28178927]

20. Prescott HC, Chang VW. Overweight or obese BMI is associated with earlier, but not later survival after common acute illnesses. BMC Geriatr. 2018;18(1):42. [PubMed: 29409463]

21. Brilleman SL, Wolfe R, Moreno-Betancur M, et al.Associations between community-level disaster exposure and individual-level changes in disability and risk of death for older Americans. Soc Sci Med. 2017;173:118-125. [PubMed: 27960126] 


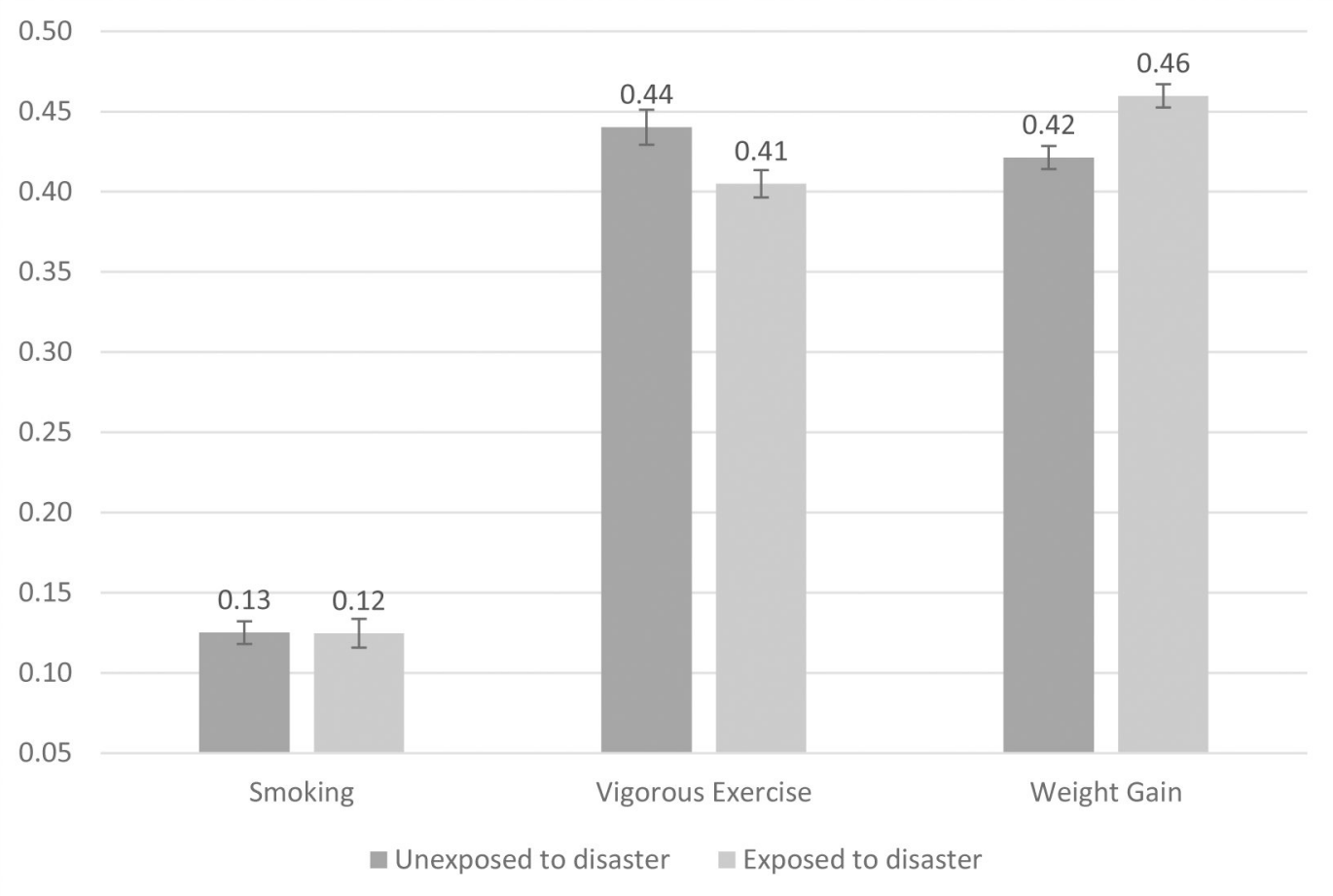

Figure 1.

Predicted Probabilities of Health Risk Behavior and Disaster Exposure from Fully Adjusted Model. 
Table 1.

Unadjusted and Adjusted Odds Ratios and Relative Risk Ratios of Health Risk Behaviors and Exposure to Disaster

\begin{tabular}{|c|c|c|c|}
\hline Outcomes & Point Estimate & $\mathbf{( 9 5 \%}$ CI $)$ & $\boldsymbol{P}$ Value \\
\hline Physical Activity \\
\hline Unadjusted & OR $=0.89$ & $(0.84-0.95)$ & .001 \\
\hline Adjusted & OR $=0.84$ & $(0.79-0.89)$ & $<.001$ \\
\hline Weight Gain (Base Outcome $=$ No Weight Change) \\
\hline Unadjusted & RRR $=1.19$ & $(1.11-1.27)$ & $<.001$ \\
\hline Adjusted & RRR $=1.21$ & $(1.13-1.30)$ & $<.001$ \\
\hline Weight Loss (Base Outcome $=$ No Weight Change $)$ \\
\hline Unadjusted & RRR $=1.03$ & $(0.97-1.10)$ & .32 \\
\hline Adjusted & RRR $=1.05$ & $(0.98-1.12)$ & .16 \\
\hline Smoking & OR $=0.96$ & $(0.87-1.06)$ & .40 \\
\hline Unadjusted & OR $=0.99$ & $(0.91-1.08)$ & .92 \\
\hline Adjusted &
\end{tabular}

Note: Odds ratios (OR) are for logistic regression, relative risk ratios (RRR) for multinomial logistic regression. 